Revista de Matemática: Teoría y Aplicaciones 2008 15(1) : 13-26

CIMPA - UCR ISSN: 1409-2433

\title{
NAUTELIA: SISTEMA AUTOMATIZADO PARA EL RUTEO NÁUTICO METEOROLÓGICO
}

\author{
Yovany Cordero Hernández* Karelia Fernández Castillo ${ }^{\dagger}$ \\ Marta L. Baguer Díaz-Romañach ${ }^{\ddagger} \quad$ Anneris CALnicK ${ }^{\S}$ \\ ISRAEL BORRAJEROS $\Phi^{\top}$
}

Recibido/Received: 24 Jul 2007; Aceptado/Accepted: 31 Oct 2007

\begin{abstract}
Resumen
Para el capitán de un buque es muy importante contar con una propuesta de ruta que le permita llegar al puerto deseado en el menor tiempo posible considerando las condiciones meteorológicas y oceanográficas imperantes. Para encontrar esta ruta de tiempo mínimo se necesita de una previa estimación de las variables meteorológicas para todos los días que comprende la travesía, ya que estas influyen directamente en la reducción de la velocidad del buque. Esta estimación se realiza utilizando los valores de pronósticos obtenidos de Internet.

Para obtener una ruta lo suficientemente rápida se deben transformar los datos de pronósticos tal que puedan ser utilizados posteriormente en los cálculos de la velocidad de la embarcación. Luego, encontrar modelos que describan la influencia de cada una de las variables meteorológicas en la velocidad del buque para una cierta coordenada y un tiempo dado y deducir una expresión que permita calcular el tiempo de travesía en dependencia de la ruta programada y la velocidad puntual del buque a lo largo de la misma. Por último, se debe optimizar el tiempo de travesía, básicamente variando la ruta planificada a partir de la aplicación de métodos de optimización.
\end{abstract}

Palabras clave: Programación no lineal, interpolación, splines, ecuaciones diferenciales ordinarias, algoritmos genéticos.

\footnotetext{
*Departamento de Matemática Aplicada, Facultad de Matemática y Computación, Universidad de la Habana, San Lázaro y L, La Habana CP 10400, Cuba. E-Mail: yovany@matcom.uh.cu

${ }^{\dagger}$ E-Mail: karelia_fdez@yahoo.es

${ }^{\ddagger}$ Misma dirección que Y. Cordero. E-Mail: mbaguer@matcom.uh.cu

${ }^{\S}$ Instituto de Meteorología, La Habana, Cuba. E-Mail: anneris.calnick@insmet.cu

『Misma dirección que A. Calnick. E-Mail: israel.borrajeros@insmet.cu
} 
For the captain of a ship, it is very important to have a proposal of the route that allows him to reach the desired destiny as soon as possible, taking into account weather conditions. In order to find such a route a former estimation of meteorological variables for every single day of the journey is needed, because those variables have a direct influence in the speed of the ship. This estimation is done using the weather forecast, obtained from the Internet.

To find a route fast enough one must transform the weather forecast data so that it can be used afterwards for computing the speed. Besides, it is necessary to build models that describe the influence of each one of the meteorological variables in the speed of the ship for a certain place and time and estimate the journey time for a specific route, depending on the ships characteristics. Finally, the sailing time is optimized, basically changing the initial route using optimization methods.

Keywords: Nonlinear programming, interpolation, splines, ordinary differential equations, genetic algorithms.

Mathematics Subject Classification: 65K05, 65D05, 65D07, 65L06, 92D99, 68T20.

\section{Introducción}

El Ruteo Náutico Meteorológico no es más que el procedimiento mediante el cual se obtienen rutas marítimas "óptimas" en función de las condiciones meteorológicas y oceanográficas para un determinado buque, teniendo en cuenta las características técnicas de este [Cal]. Para el capitán de un buque es de gran importancia contar con este tipo de servicio pues le permite llegar al puerto destino en un menor tiempo, sin embargo, se pueden encontrar en el mundo muy pocas empresas que brindan este servicio y las que existen son grandes consorcios que venden sus servicios a precios impagables para la mayoría de los países del Tercer Mundo. Cuba ha buscado alternativas para brindarle dicho servicio a su flota de buques.

A partir del año 1987 el Centro de Meteorología Marítima del Instituto de Meteorología de Cuba comienza a brindar el servicio de ruteo con gran eficacia realizando todos los cálculos necesarios manualmente. En el año 1999 ve la luz el software ROUTES, una herramienta desarrollada en $\mathrm{C}++$ por Anneris Calnick e Israel Borrajeros. Dicho software brinda una serie de facilidades apreciables para el usuario, y los buenos resultados en su utilización han sido ya demostrados.

El sistema NAUTELIA introduce otros métodos numéricos y sobre todo combina, en el proceso de obtención de la "ruta óptima", estrategias deterministas y no deterministas.

Esto, junto a la realización en un lenguaje más moderno, hacen del mismo una herramienta muy útil y flexible para la actualización y el reúso.

\section{Presentación del problema}

El problema a resolver, visto de la manera más dinámica y simple posible, sería: sugerir al mando de un buque -que debe emprender un viaje desde un puerto de salida hasta un cierto destino- una ruta inicial que minimice en lo posible el tiempo de travesía, dando 
las facilidades de modificar la ruta así llamada "óptima" calculada inicialmente, a medida que el barco cumpla distintas etapas del recorrido. La "ruta óptima", desde el punto de vista matemático, no está bien definida ya que se trabaja con datos de pronóstico en las diferentes etapas de su obtención las cuales se exponen a continuación.

\section{Obtención de los valores puntuales para las variables me- teorológicas y oceanográficas}

Las condiciones meteorológicas y oceanográficas (altura y dirección de los campos de ola y dirección e intensidad de la corriente respectivamente) existentes en el período en el que transcurre la travesía tienen una influencia decisiva en la reducción de la velocidad del buque. Por tal motivo es de vital importancia poder conocer el valor de estas variables en cualquier par de coordenadas-tiempo. Sin embargo, los datos que se utilizan en algunos casos no cubren todo el intervalo de tiempo que puede durar la travesía y están dados de manera discreta para coordenadas que forman una rejilla rectangular de datos y para determinados tiempos en intervalos equidistantes. Por lo cual requieren de un procesamiento inicial.

En el caso de los valores de intensidad y dirección de la corriente se cuenta con los pilots charts, cartas mensuales que brindan estos datos para cada época del año. Son, en definitiva, rejillas de valores correspondientes al día medio de cada mes, con una resolución de $2.5^{\circ}$ de latitud y longitud. Para facilitar cálculos posteriores se calculan las componentes longitudinales y latitudinales de la intensidad de la corriente, para cada par de coordenadas-tiempo existente, a partir de los datos originales y estos son los valores a interpolar:

$$
\begin{aligned}
& V_{c x}=l_{i j k} \cdot \cos \left(D_{i j k}\right) \\
& V_{c y}=l_{i j k} \cdot \sin \left(D_{i j k}\right)
\end{aligned}
$$

donde $V_{c x}$ es la componente longitudinal, $V_{c y}$ la componente latitudinal, $l_{i j k}$ la intensidad de la corriente, y $D_{i j k}$ la dirección de la corriente.

Luego, para obtener los valores puntuales primeramente se escogen tres matrices consecutivas, donde la primera es la correspondiente al día medio anterior al inicio de la travesía y se calculan matrices con una resolución menor $-1^{\circ}$ de latitud y longitud y cada 6 horas- utilizando interpolación tridimensional por splines [Nur]. Estas matrices se almacenan en memoria y cuando se requiere el valor de estas variables en un par coordenadas-tiempo dado se realiza una interpolación lineal en tres dimensiones [Nur].

Los valores de altura y dirección de los campos de ola son generados por un modelo que describe la generación de los campos de olas por la acción del viento y que utiliza como datos de entrada la velocidad y dirección del viento. Estos datos a su vez son obtenidos de un sitio en Internet. Finalmente contamos con rejillas tridimensionales de latitud, longitud y tiempo, con una resolución de $1^{\circ}$ de latitud y longitud y cada 3 horas. Para obtener los valores puntuales se realiza una interpolación lineal tridimensional [Nur]. 


\section{Cálculo de la velocidad reducida del buque teniendo en cuenta las condiciones meteorológicas}

Una vez conocidos los valores de altura y dirección de los campos de olas e intensidad y dirección de las corrientes marinas para una coordenada determinada en un momento dado, se hace necesario encontrar una expresión que permita, a partir de los valores antes mencionados, calcular la velocidad reducida del buque.

Primero se calcula la variación de la velocidad por el efecto de las variables meteorológicas, utilizando la formulación de Krasiuk [Cal], la cual además tiene en cuenta el desplazamiento del buque y la velocidad pactada con el capitán.

$$
V=V_{0}-(0.745 \cdot h-0.275 \cdot q \cdot h) \cdot\left(1.0-1.35 \cdot 10^{-6} \cdot D \cdot V_{0}\right)
$$

donde $V$ es la velocidad resultante del buque al considerar la influencia de los campos de olas, $V_{0}$ la velocidad pactada del buque, $h$ la altura de la ola, $q$ el ángulo de incidencia de la ola, y $D$ el desplazamiento del buque (equivalente a su peso).

Una vez calculada la influencia de los campos de olas en la reducción de la velocidad, se debe considerar la acción de las corrientes marinas sobre el movimiento del buque. Para la coordenada y el tiempo específico, son obtenidas las componentes tangencial y transversal de la corriente a partir de (4) y (5). La primera es sumada algebraicamente a la velocidad (6). En el caso de la segunda, se incorpora teniendo en cuenta el alargamiento de trayectoria provocado; esto es, al incidir la corriente en el movimiento del buque, provoca un cambio en su rumbo que debe ser corregido para mantener la dirección original (7) [Cal].

$$
\begin{gathered}
V_{\uparrow}=V_{c y} \cdot \cos (A z)-V_{c x} \cdot \sin (A z) \\
V_{\leftrightarrow}=V_{c x} \cdot \cos (A z)+V_{c y} \cdot \sin (A z)
\end{gathered}
$$

donde $V_{\uparrow}$ es la componente tangencial de la corriente, $V_{\leftrightarrow}$ la componente transversal de la corriente, $V_{c x}$ la componente longitudinal de la corriente, $V_{c y}$ la componente latitudinal de la corriente, y $A z$ el acimut de la dirección del buque.

El acimut o ángulo de dirección del barco con respecto al norte (girando en el sentido de las manecillas del reloj como se muestra en la Figura 1), puede ser hallado, dada una ruta ortodrómica y un punto cualquiera en la misma, de manera unívoca (12).

$$
\begin{gathered}
V_{r}^{1}=V_{r}^{0}+V_{\uparrow} \\
V_{r}=V_{r}^{1} \cdot\left(\arctan \left(\frac{V_{\leftrightarrow}}{V_{r}^{1}}\right)\right)
\end{gathered}
$$

donde $V_{r}^{1}$ es la velocidad resultante de la suma algebraica de la velocidad de la corriente tangencial y la velocidad reducida por olas, $V_{r}^{0}$ la velocidad reducida por olas, y $V_{r}$ la velocidad reducida tomando en cuenta corrientes y olas. 
El valor computado en (sumCorr) describe finalmente el comportamiento de la velocidad del buque, teniendo en cuenta las condiciones meteorológicas, en una coordenada y tiempo determinado.

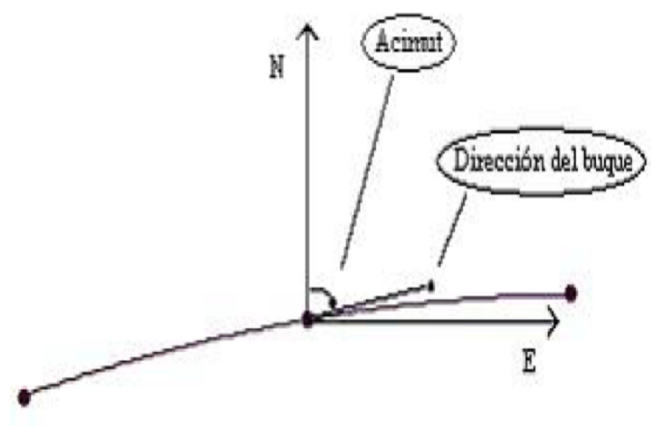

Figura 1: Acimut o dirección del buque.

\section{Cálculo de la trayectoria de círculo máximo entre dos pun- tos}

El próximo paso es, determinar cuáles son los puntos por los que el buque transitará, de acuerdo con la ruta que debe seguir. Se define una ruta como un conjunto ordenado de puntos en el mapa, donde cada punto y su consecutivo se encuentran unidos por una ruta de círculo máximo (Figura 2). Por tanto, si se tiene la forma de conocer entre dos coordenadas cualesquiera, cual es el lugar geométrico de los puntos que se encuentran sobre la ruta ortodrómica que los une, entonces, se podrá determinar exactamente todas las coordenadas de la trayectoria de la embarcación.

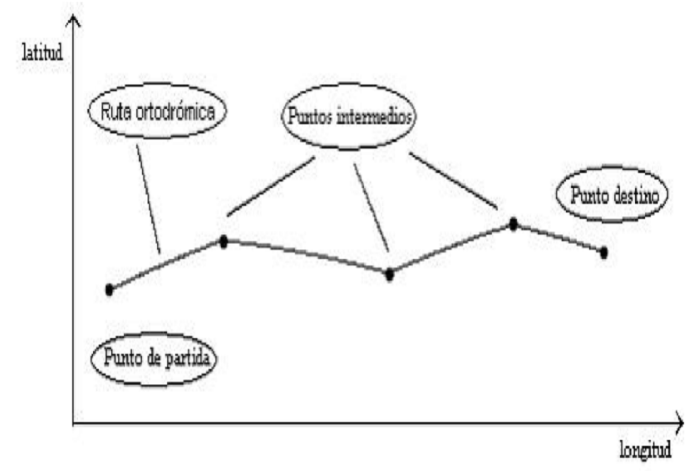

Figura 2: Posible ruta a seguir por el buque.

Para esto, se utilizan ecuaciones que se basan en calcular primeramente, un vértice en 
la trayectoria ortodrómica a partir del cual se hallan el resto de los puntos (Figura 3). Este primer paso se logra a partir de las ecuaciones:

$$
\begin{gathered}
\tan (\Delta \operatorname{long}(\angle V T))=\tan (\text { lat } F) \cdot \cot (\text { Lat } T) \cdot \csc (\Delta \operatorname{long}(\angle F T)-\cot (\Delta \text { long }(\angle F T))) \\
\cot (\text { lat })=\cot (\text { lat } F) \cdot \cos (\Delta \text { long }(\angle F V)
\end{gathered}
$$

donde $F$ es el punto inicial de la trayectoria, $T$ el ppPunto final de la trayectoria, y $V$ el vértice de la ruta de círculo máximo entre $F$ y $T$.

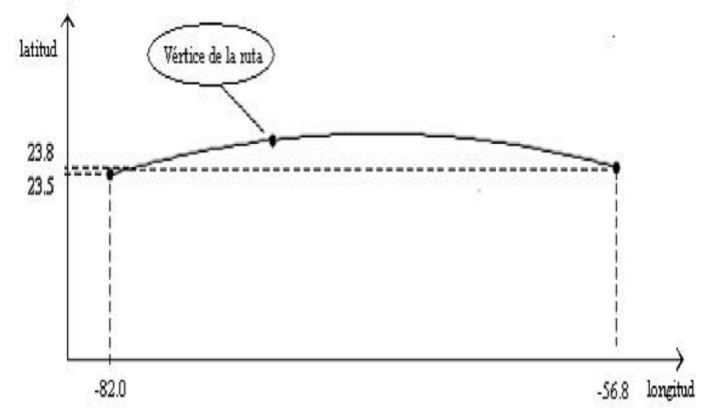

Figura 3: Ruta ortodrómica entre dos puntos

Téngase en cuenta que $\Delta l o n g(\angle V T)$ no se refiere a otra cosa que a la resta de la longitud del punto $V$ y la longitud del punto $T$. Utilizando (9) queda calculada la latitud de $V$, a partir de la longitud ya calculada.

Con el vértice de la ruta es posible obtener un punto cualquiera utilizando la ecuación (10).

$$
\tan (\text { lat } P)=\tan (\text { lat } V) \cdot \cos (\Delta \operatorname{long}(\angle V P)
$$

donde $P$ es el punto sobre la ruta otodrómica que se desea encontrar.

La longitud de $P$ puede ser cualquiera que se encuentre en el intervalo que conforman la longitud del punto inicial y final de la ruta.

Es necesario analizar cómo obtener otros valores importantes que se utilizan para darle solución al problema; a saber, la distancia de la ruta ortodrómica entre dos puntos (11) y el ángulo de trayectoria o acimut de la embarcación en un punto dado (12), utilizado en el cálculo de la velocidad reducida.

$$
\begin{gathered}
\text { dis }=\frac{\text { Rad }}{1,852} \cdot \arccos (\sin (\text { lat } I) \cdot \sin (\text { lat } F)+\cos (\text { lat } F) \cdot \cos (\Delta l o n g(\angle I F))) \\
\cos (\text { Angle })=\frac{\sin (\text { lat } F)-\sin (\text { lat } I) \cdot \cos \left(\frac{\text { dis }}{\text { Rad }}\right)}{\cos (\text { latI }) \cdot \sin \left(\frac{\text { dis }}{\text { Rad }}\right)}
\end{gathered}
$$

donde $R a d$ es el radio medio de la tierra en Km, I el extremo inicial de la trayectoria ortodrómica, es el punto sobre el que se calcula el acimut en el caso del ángulo, $F$ el 
extremo final de la trayectoria ortodrómica, un punto cualquiera sobre la ruta en el cálculo del ángulo, Dis es la d Distancia entre $I$ y $F$ en Km, y Angle el ángulo de trayectoria del buque.

\section{Cálculo del tiempo de travesía a lo largo de una ruta ortodrómica}

Hasta ahora se conocen cuáles son los puntos del recorrido del buque según una ruta especificada. También es posible determinar, para cada uno de estos puntos, el valor de la velocidad del buque en cualquier tiempo deseado. Sería deseable, entonces, estimar el tiempo que demora un buque al transitar por una ruta específica.

El cálculo del tiempo se realiza de manera puntual a través de la solución de una ecuación diferencial no lineal como la (13)

$$
\frac{d t}{d s}=\frac{1}{v(s, t)}
$$

donde: $v(s, t)$ es la velocidad reducida del buque tomando en cuenta las olas y las corrientes (7), y $s$ los parámetros que se utilizan para obtener la velocidad en un punto y un momento determinado; es decir, longitud y latitud del punto y acimut del barco en ese punto.

Para resolver esta ecuación diferencial por métodos numéricos se explora más de una alternativa, con el fin de que el usuario tenga la flexibilidad de escoger una u otra para realizar los cálculos.

La primera de las estrategias consiste en utilizar una combinación del método RungeKutta de segundo orden - para las primeras iteraciones en cada intervalo de la rutacon un esquema predictor-corrector [Nur]. La otra variante es utilizar siempre el método de Runge-Kutta de cuarto orden [Nur] en todas las iteraciones. Para esta variante, no se toma un tamaño de paso fijo, sino que se asume una estrategia de paso variable para lograr que el error, estimado por doble cómputo, se mantenga dentro de un rango deseado. [Nur]

El tiempo de duración de la travesía se puede calcular sumando los tiempos calculados para cada par de puntos consecutivos que conforman la ruta. Esta idea se expresa en (14).

$$
T=\sum_{i} t_{i}
$$

donde $t_{i}$ es el valor del tiempo calculado para el $i$-ésimo segmento, que depende a su vez del tiempo transcurrido al iniciar este tramo de travesía y de los puntos inicial y final del mismo. Luego:

$$
T=T\left(\operatorname{lat}_{i}, \operatorname{lon}_{i}\right)
$$

donde $i$ representa el número del nodo. 


\section{Optimización del tiempo de travesía}

Al ser el tiempo función de las coordenadas de los distintos puntos de la ruta, es posible formular un problema de optimización no lineal sin restricciones (16) donde las variables son en efecto dichas coordenadas.

$$
\min T\left(\text { lat }_{i}, \operatorname{lon}_{i}\right)
$$

donde $l a t_{i}$ es la latitud del $i$-ésimo punto no fijo de la trayectoria, y $l o n_{i}$ la longitud del $i$-ésimo punto no fijo de la trayectoria.

Las restricciones del tipo $-180^{\circ} \leq$ lat $_{i}$, lon $_{i} \leq 180^{\circ}$ no son necesarias ya que estos valores son utilizados en las fórmulas como argumentos de funciones trigonométricas, que toman los mismos valores para ángulos coterminales. Tampoco se consideran restricciones del tipo $\operatorname{lon}_{i} \leq \operatorname{lon}_{j} \forall i \leq j$, dándole al buque la flexibilidad suficiente de atrasar un poco su recorrido si, siguiendo esa trayectoria, se encuentra condiciones meteorológicas favorables que agilicen el mismo (aunque la práctica indica que es muy poco probable que esto ocurra).

El problema (16) puede resolverse mediante la aplicación de métodos de optimización deterministas para problemas sin restricciones [Lue]. Además, con el objetivo de experimentar en la utilización de otro tipo de técnicas para hallar, por ejemplo, una buena aproximación inicial o recuperarnos de un mínimo local obtenido por esta vía, se ha diseñado un algoritmo genético.

\subsection{Una aproximación inicial}

En cualquier caso, lo mismo si se pretende optimizar el tiempo utilizando un algoritmo determinista o una heurística, se necesita de un punto de partida; en este caso una ruta inicial a mejorar. En ambos casos sería deseable que dicha ruta fuera la mejor posible, y entiéndase por mejor, según el criterio de optimización, aquella que necesite de un tiempo menor para ser recorrida. De esa manera, se asegura una convergencia más rápida al óptimo. Sin embargo, no existe un único criterio de cómo escoger esa primera ruta. En nuestro caso se escogerá aquella de menor distancia con una distribución de puntos intermedios equidistantes en el tiempo. Esta opción no es muy descabellada, teniendo en cuenta que en condiciones de calma meteorológica es la más rápida.

\subsection{Optimización determinista}

Para resolver el problema de optimización (16) se utiliza un BFGS [Lue] que busca la dirección de descenso. Considerando lo complicado que sería encontrar una expresión analítica para el cálculo del tiempo (tómese en cuenta que depende de valores que se obtienen a partir de la integración de una ecuación diferencial, que involucra interpolaciones por splines, interpolaciones lineales, etc.) se aproxima el gradiente utilizando diferencias divididas, específicamente, diferencias centrales [Stoe]. El tamaño del paso debe ser calculado por un método que sea bastante eficiente en tiempo de cómputo. Además, no debe utilizar información sobre la derivada de la función para evitar los errores de aproximación 
al calcular la misma utilizando diferencias finitas. Esta es la razón por la que se utiliza la Regla de Armijo [Lue], cuyos resultados en la práctica son bastante buenos.

\subsection{Optimización no determinista}

A partir de la descripción del funcionamiento de un algoritmo genético [Coe] se deducen los componentes básicos a tener en cuenta para resolver un problema de optimización con este tipo de algoritmo:

1. Una representación de soluciones potenciales del problema.

2. Una forma de crear una población inicial de soluciones potenciales.

3. Una función de evaluación que juega el papel de ambiente, calificando a las soluciones generadas en términos de su aptitud.

4. Operadores genéticos que alteran la composición de los descendientes.

5. Valores para los diversos parámetros utilizados por el algoritmo genético.

6. Un criterio de parada del algoritmo.

\subsubsection{Representación}

Un individuo será una lista de puntos (longitud, latitud), a través de los cuales debe pasar

el buque durante su travesía hacia el puerto destino. Estos a su vez son los genes de dicho individuo.

\subsubsection{Generación de la población inicial}

Una población será un conjunto de posibles rutas a seguir por el buque. Cada individuo de la población inicial se generará utilizando como patrón la ruta que constituye la aproximación inicial, modificándose aleatoriamente las componentes de cada punto dentro de un rango $\left(\operatorname{lon}_{i} \pm 1^{\circ}\right.$, lat $\left._{i} \pm 3^{\circ}\right)$.

\subsubsection{Función de aptitud}

Como el principal objetivo que se persigue es minimizar el tiempo de travesía, la función de aptitud será (14).

\subsubsection{Operadores genéticos}

Se adaptaron a este problema algunos de los operadores de selección, cruzamiento, mutación y reemplazamiento existentes, como son: mutación uniforme y no uniforme, cruzamiento uniforme y por dos puntos, selección mediante ruleta y torneo probabilístico, entre otros. 


\subsubsection{Valores predeterminados para los parámetros del algoritmo}

Aún cuando el software brinda la posibilidad de modificar todos los parámetros, es preciso definir los valores que se asumen como predeterminados, en este caso el tamaño de la población es 15 , las probabilidades de cruzamiento y mutación son ambas 0.7 , el porcentaje de reemplazamiento es del $90 \%$, se asumen 60 generaciones y para la convergencia un $50 \%$. Es preciso destacar que esta selección está en correspondencia con los buenos resultados prácticos obtenidos en la mayoría de las corridas de experimentación realizadas.

\subsubsection{Criterios de parada}

Como criterios de parada se utilizaron: detener el algoritmo cuando se haya alcanzado un número máximo de iteraciones (que el usuario puede indicar) o cuando la población se haya estabilizado, es decir, cuando un porcentaje determinado de los mejores individuos de las últimas $n$ generaciones tengan similar aptitud.

\section{Algunos resultados de la experimentación con Nautelia}

\subsection{Validación de la obtención de la ruta óptima}

Los métodos de optimización son quizás, los más importantes a la hora de obtener la ruta óptima. Además, lograr su funcionamiento correcto es tarea compleja, teniendo en cuenta que utilizan el resto de los métodos numéricos y necesitan del ajuste de múltiples parámetros, sobre todo, los algoritmos genéticos. Es por esto que la validación de los mismos es esencial en la demostración del buen funcionamiento del software.

Para la primera experimentación se utilizaron las matrices de altura cero generadas para comprobar el tiempo de travesía, motivados por el hecho de que en condiciones de mar en calma la velocidad del buque no sufre variaciones. Esto provoca que la ruta óptima de un punto en el mapa a otro sea la ruta de círculo máximo u ortodrómica que los une.

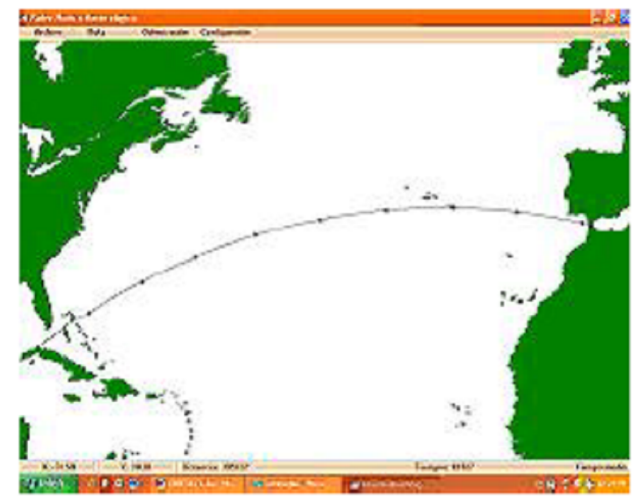

Figura 4: Ruta óptima para condiciones de mar tranquila (ortodrómica).

A continuación se muestra la aproximación inicial utilizada en esta experimentación y la ruta obtenida al finalizar el proceso de optimización. 


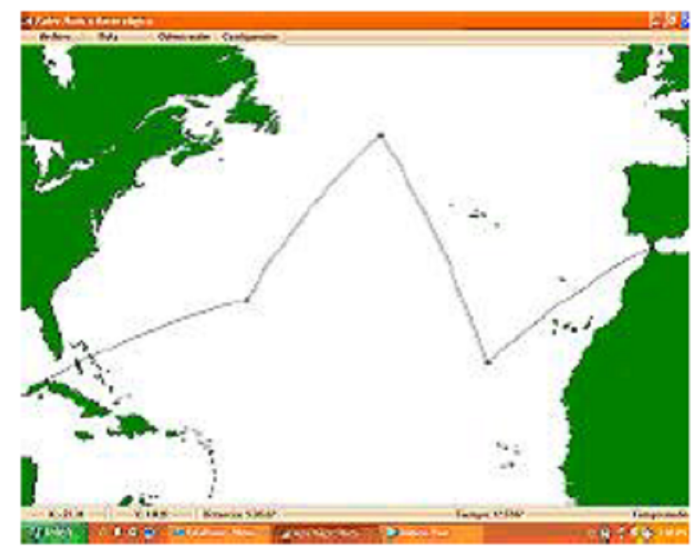

Figura 5: Ruta a optimizar.

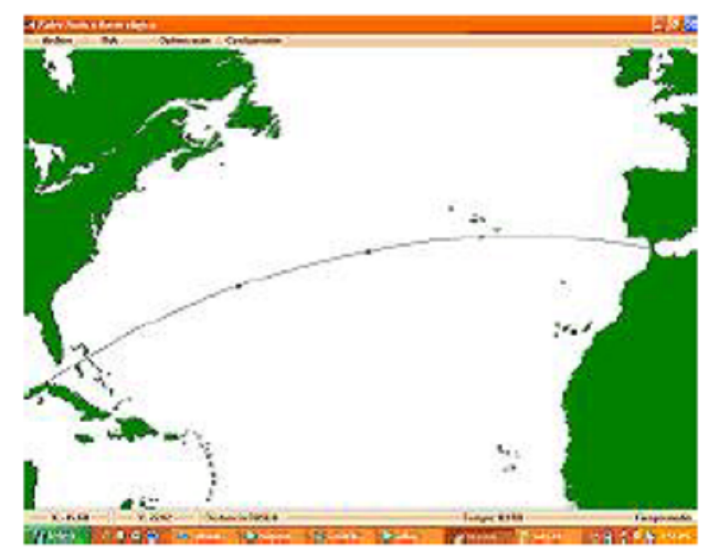

Figura 6: Ruta optimizada por Nautelia.

En la ruta inicial mostrada en la Figura 5, el tiempo de travesía es de 12.3747 días, y la distancia a recorrer es de 5345.87 millas náuticas. Al aplicarle un algoritmo de optimización (esta corrida específica corresponde a la heurística) se obtuvo la ruta de la Figura 6, cuya longitud es de 3958.4 y es recorrida en 9.1628 días. Una simple comparación con la ruta ortodrómica que une a estos puntos en la Figura 4 revela una diferencia de sólo 0.08 millas náuticas y 0.0001 días (¡0.1 min!) entre una y otra.

Un segundo experimento consistió en considerar condiciones de mar en calma y penalizar una zona del océano - por la cual pasaba una ruta ortodrómica - con condiciones adversas para el avance del buque, a saber, con olas de 20 pies de altura y dirección $90^{\circ}$, contrarias al movimiento de la embarcación. Se escogió la ruta ortodrómica y el resto del mar en calma para asegurar que si existe cualquier variación al optimizarla, es necesariamente provocada por la zona penalizada.

En la Figura 7 se muestran la ruta ortodrómica inicial y los campos de olas, cuyas direcciones se corresponden con el sentido de las flechas y la zona penalizada se encuentra coloreada con un rojo intenso. En la Figura 8 aparece la ruta optimizada.

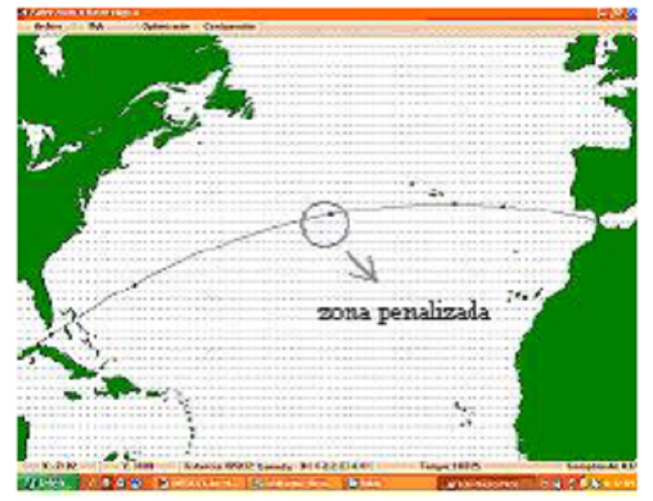

Figura 7: Ruta con zona penalizada.

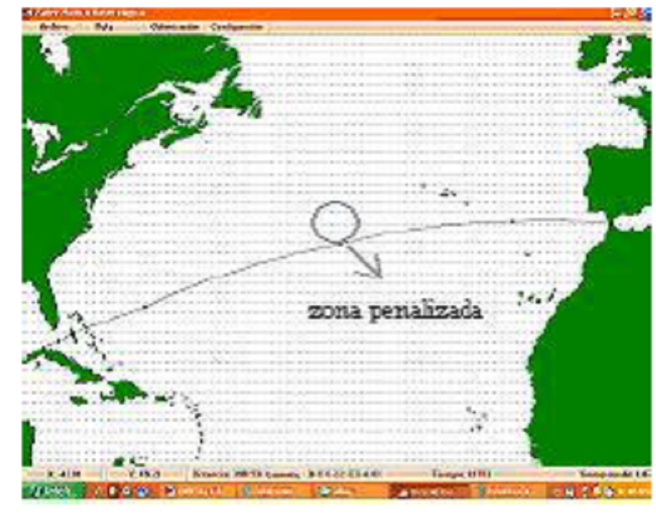

Figura 8: Ruta optimizada.

Nótese que el algoritmo decidió desviar el rumbo del buque de la zona penalizada, 
otorgándole aquella ruta de menor distancia (verificado al mover el punto durante la experimentación) que mantuviera las condiciones de mar en calma. De esta manera el tiempo de la ruta inicial de 10.01 días fue disminuido hasta 9.1703 días. Se puede observar que en comparación con los tiempos y longitudes para la ruta ortodrómica con condiciones de mar en calma en todo el mapa (ver Figura 4) la longitud y el tiempo de la ruta aumentaron 2 millas náuticas y 1 minuto respectivamente.

Estos dos ejemplos no constituyen los únicos experimentos que corroboran la buena aproximación de la ruta óptima. En muchas ocasiones se han obtenido óptimos que difieren bastante de la ruta de menor distancia, sin embargo, al visualizar el recorrido del buque y los campos de olas simultáneamente se ha podido observar cómo el algoritmo ha obtenido una ruta en la que el buque encuentra generalmente los campos de olas en la dirección de su recorrido, lo que provoca un aumento considerable de su velocidad.

\subsection{En busca de una estrategia eficiente}

En algunas corridas de prueba se detectó que el óptimo obtenido por una de las estrategias difería en más de una hora del óptimo hallado por la otra. En otros casos, aquella estrategia que resultó ser peor en las corridas señaladas arrojó mejores resultados.

De manera general, se comprobó que muchas veces, cuando el valor del tiempo para la aproximación inicial difiere bastante del valor correspondiente a la ruta óptima la aplicación del algoritmo genético reporta mejores resultados. Por otra parte si la ruta se encuentra subdividida en una cantidad grande de intervalos, la aplicación del BFGS generalmente garantiza mejores óptimos. Luego, ¿por qué no experimentar con la combinación de ambas estrategias?

Siguiendo esta idea se realizaron numerosos experimentos que alternaban la utilización de ambos tipos de métodos, provocando una mejora de la función objetivo en casi la totalidad de los casos. Para ilustrarlo se presenta la siguiente secuencia de aplicaciones alternadas de estos métodos en una de las corridas experimentales.

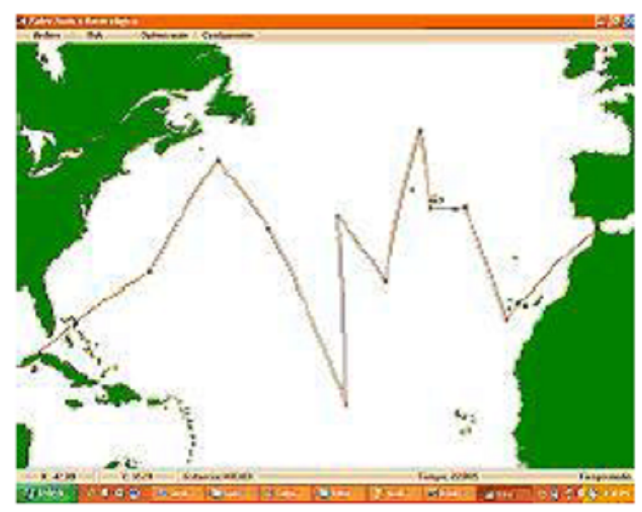

Figura 9: Ruta inicial.

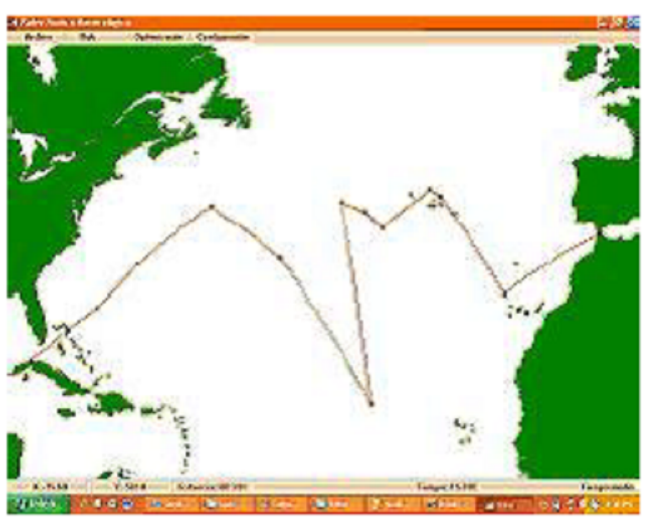

Figura 10: Ruta obtenida por determinista.

Las condiciones meteorológicas fueron tomadas para mar tranquila de forma tal que tuviéramos conocimiento del óptimo del problema (representado en la Figura 4). Resultó 


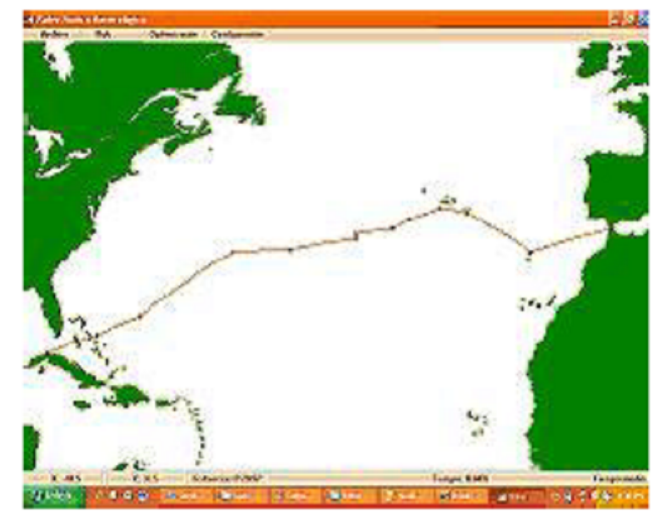

Figura 11: Ruta corregida por genético.

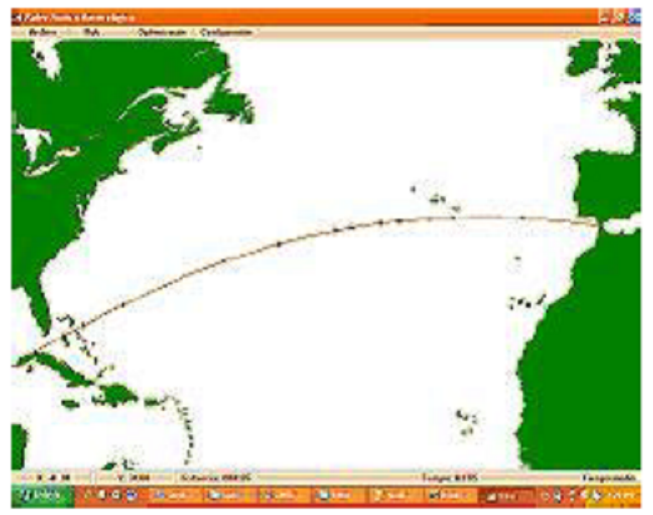

Figura 12: Ruta corregida por determinista.

que ninguna de las estrategias puras fue capaz de resolver el problema de manera eficiente. $\mathrm{Al}$ aplicar BFGS se redujo el tiempo de la ruta inicial de 22.69 días (Figura 9) a 15.31 días (Figura 10). Sin embargo, la ruta óptima podía ser salvada en tan sólo 9.16 días. ¿Qué ocurrió?

La mayoría de los métodos de optimización no lineal deterministas utilizan como condición de parada la nulidad del gradiente en el punto de la iteración actual. En el caso del tiempo, que constituye la función objetivo, el gradiente no puede calcularse de manera exacta (salvo el error de redondeo), sino que es aproximado por diferencias finitas. El error de truncamiento en que se incurre al aproximar el mismo puede traer como consecuencia que sea "reconocido" un óptimo local inexistente, provocando la parada del algoritmo. Otra causa posible puede estar dada porque la función tenga, efectivamente, más de un mínimo local. De esta manera, si el primer mínimo local alcanzado no es el mínimo global de la función, el algoritmo para sin llegar a obtener la ruta de menor tiempo.

Tanto en el primer caso como en el segundo una posible solución sería lograr de alguna manera sacar al algoritmo de este punto de "falso óptimo", de forma tal que el nuevo punto provoque un decrecimiento en la evaluación de la función objetivo. De esta manera se estaría evitando volver a alcanzar dicho punto al continuar con la aplicación del algoritmo, ya que este sólo se mueve por direcciones de descenso. La manera ideal de lograrlo es aplicando un algoritmo de optimización que no utilice evaluaciones del gradiente, justo el caso de los algoritmos genéticos.

Como muestra la Figura 11 la aplicación del genético a la ruta obtenida por el BFGS provocó una mejora sustancial de la solución, sin embargo debido a que estos algoritmos no obtienen soluciones exactas y quizás porque la ruta que inicializó el algoritmo se encontraba aún distante del óptimo, la ruta devuelta aún podía ser mejorada. Una última aplicación del algoritmo determinista (Figura 12) reportó una increíble aproximación al óptimo. Se constató una diferencia de tan sólo 2 millas náuticas y 10 minutos con respecto a la longitud y el tiempo respectivamente correspondientes al óptimo. 
26 Y.Cordero-K.FernándeZ-M.BAGUer-A.CALniCK-I.Borrajeros Rev.Mate.Teor.Aplic. (2008) 15(1)

\section{Referencias}

[Coe] Coello, C. (2003) Introducción a la Computación Evolutiva. Lab. Nacional de Informática Avanzada, México.

[Cal] Calnick, A. (2003) Sistema Automatizado para el Pronóstico de Rutas Náuticas en Función de las Condiciones Meteorológicas. Tesis de Doctorado, Universidad de la Habana, La Habana.

[Lue] Luenberger, D. (1984) Introduction to Linear and Nonlinear Programming. Adisson Wesley.

[Nur] Press, W.H.; Flannery, B.P.; Teulolsky, S.A.; Vetterling, W.T. (1992) Numerical Recipes in C: The Art of Scientific Computing. Cambridge University Press, EE.UU.

[Stoe] Stoer, J.; Bulirsch, R. (2005) Numerische Mathematik. Springer Verlag, Berlin. 